\title{
Correction to: Drug tolerability and reasons for discontinuation of seven biologics in 4466 treatment courses of rheumatoid arthritis - the ANSWER cohort study
}

\author{
Kosuke Ebina ${ }^{1 *}$, Motomu Hashimoto², Wataru Yamamoto ${ }^{2,3}$, Toru Hirano ${ }^{4}$, Ryota Hara ${ }^{5}$, Masaki Katayama ${ }^{6}$, \\ Akira Onishi ${ }^{7}$, Koji Nagai $^{8}$, Yonsu Son ${ }^{9}$, Hideki Amuro ${ }^{9}$, Keiichi Yamamoto ${ }^{10}$, Yuichi Maeda ${ }^{4}$, Koichi Murata ${ }^{2}$, \\ Sadao Jinno ${ }^{7}$, Tohru Takeuchi ${ }^{8}$, Makoto Hirao ${ }^{1}$, Atsushi Kumanogoh ${ }^{4}$ and Hideki Yoshikawa ${ }^{1}$
}

\section{Correction to: Arthritis Res Ther \\ https://doi.org/10.1186/s13075-019-1880-4}

Following publication of the original article [1], the authors noticed that two corrections were not implemented during the production process. The original article [1] has been corrected.

In Fig. 1b, the heading should read: Adjusted survival due to lack of effectiveness

In Fig. 2b, the heading should read: Adjusted survival due to toxic adverse events

In addition, the authors would like to update the legends of Figure 1, 2, 3 and 4 to the following:

Fig. 1 Drug survival rates due to lack of effectiveness in a non-adjusted cases and $\mathbf{b}$ adjusted cases. Adjusted confounders were baseline sex, age, disease duration, concomitant prednisolone and methotrexate, and number of previously used bDMARDs. ABT = abatacept, $\mathrm{ADA}=$ adalimumab, $\mathrm{CZP}=$ certolizumab pegol, ETN = etanercept, GLM = golimumab, IFX = infliximab, TCZ = tocilizumab, bDMARDs = biological disease-modifying antirheumatic drugs

Fig. 2 Drug survival rates due to toxic adverse events in $\mathbf{a}$ non-adjusted cases and $\mathbf{b}$ adjusted cases. Adjusted confounders were baseline sex, age, disease duration, concomitant prednisolone and methotrexate, and number of previously used bDMARDs. $\mathrm{ABT}=$ abatacept, $\mathrm{ADA}=$ adalimumab, $\mathrm{CZP}=$ certolizumab pegol, ETN = etanercept, GLM = golimumab, IFX = infliximab, TCZ = tocilizumab, bDMARDs = biological disease-modifying antirheumatic drugs

Fig. 3 Drug survival rates due to remission in a nonadjusted cases and $\mathbf{b}$ adjusted cases. Adjusted confounders were baseline sex, age, disease duration, concomitant prednisolone and methotrexate, and number of previously used bDMARDs. ABT = abatacept, $\mathrm{ADA}=$ adalimumab, $\mathrm{CZP}=$ certolizumab pegol, ETN = etanercept, GLM = golimumab, IFX = infliximab, TCZ = tocilizumab, bDMARDs = biological disease-modifying antirheumatic drugs

Fig. 4. Overall drug survival rates (excluding non-toxic reasons and remission) in $\mathbf{a}$ non-adjusted cases and $\mathbf{b}$ adjusted cases. Adjusted confounders were baseline sex, age, disease duration, concomitant prednisolone and methotrexate, and number of previously used bDMARDs. $\mathrm{ABT}=$ abatacept, $\mathrm{ADA}=$ adalimumab, $\mathrm{CZP}$ = certolizumab pegol, ETN = etanercept, GLM = golimumab, IFX $=$ infliximab, $\mathrm{TCZ}=$ tocilizumab, bDMARDs = biological disease-modifying antirheumatic drugs

The publishers apologise for the errors. The corrected figures are given below:

\footnotetext{
* Correspondence: k-ebina@umin.ac.jp

1 Department of Orthopaedic Surgery, Osaka University, Graduate School of

Medicine, Osaka, Japan

Full list of author information is available at the end of the article
} 


\section{Author details}

'Department of Orthopaedic Surgery, Osaka University, Graduate School of Medicine, Osaka, Japan. ${ }^{2}$ Department of Advanced Medicine for Rheumatic Diseases, Graduate School of Medicine, Kyoto University, Kyoto, Japan.

${ }^{3}$ Department of Health Information Management, Kurashiki Sweet Hospital, Kurashiki, Japan. ${ }^{4}$ Department of Respiratory Medicine and Clinical

Immunology, Osaka University Graduate School of Medicine, Osaka, Japan.

${ }^{5}$ The Center for Rheumatic Diseases, Nara Medical University, Nara, Japan.

${ }^{6}$ Department of Rheumatology, Osaka Red Cross Hospital, Osaka, Japan.

${ }^{7}$ Department of Rheumatology and Clinical Immunology, Kobe University

Graduate School of Medicine, Kobe, Japan. ${ }^{8}$ Department of Internal Medicine (IV), Osaka Medical College, Osaka, Japan. ${ }^{9}$ First Department of Internal Medicine, Kansai Medical University, Osaka, Japan. ${ }^{10}$ Department of Medical Informatics, Wakayama Medical University Hospital, Wakayama, Japan.

Received: 15 April 2019 Accepted: 15 April 2019

Published online: 06 May 2019

\section{Reference}

1. Ebina, et al. Drug tolerability and reasons for discontinuation of seven biologics in 4466 treatment courses of rheumatoid arthritis-the ANSWER cohort study. Arthritis Res Ther. 2019;21:91 https://doi.org/10.1186/s13075019-1880-4. 

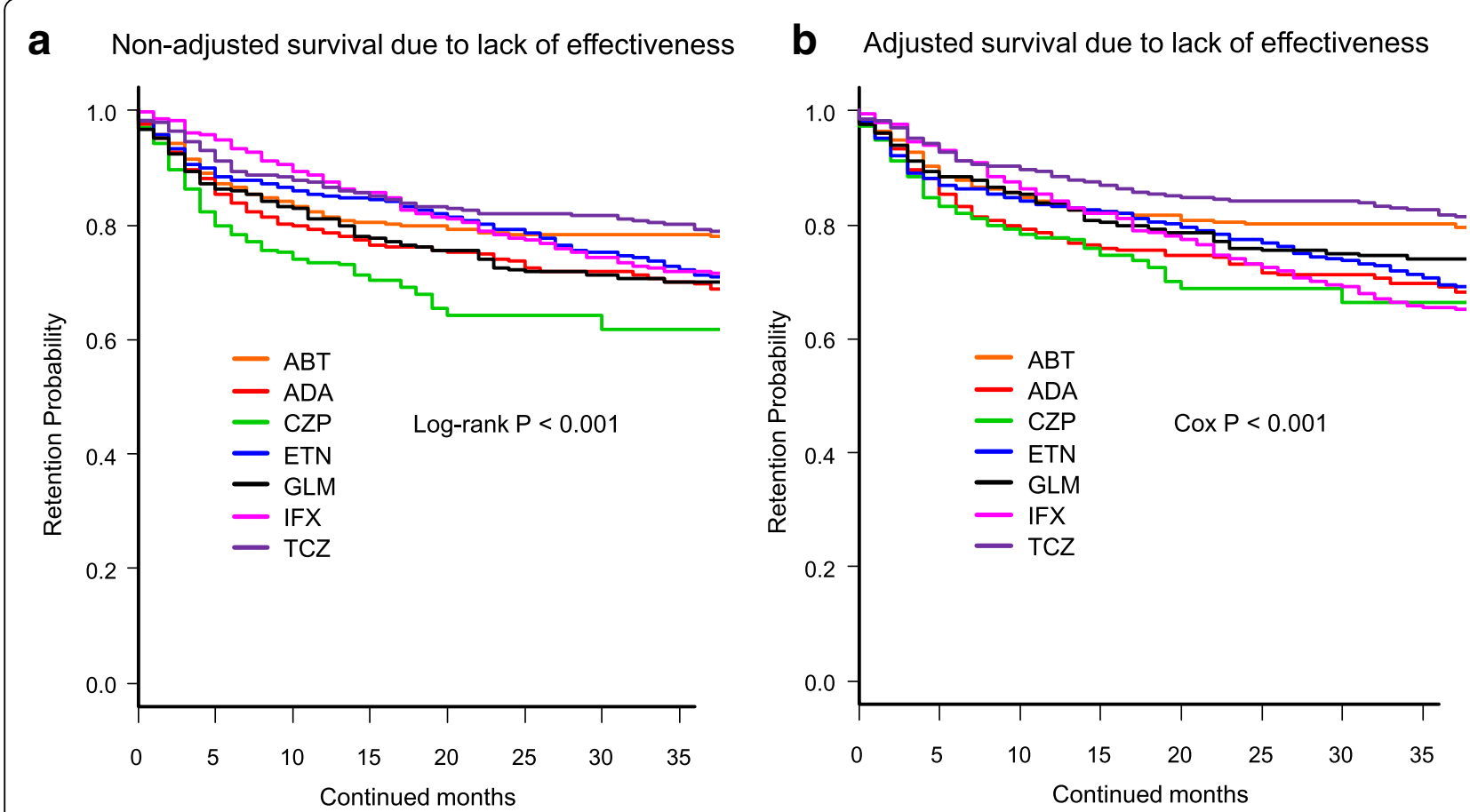

Number at risk

$\begin{array}{lllllllll}\text { ABT } & 681 & 536 & 472 & 414 & 365 & 293 & 229 & 181 \\ \text { ADA } & 558 & 419 & 361 & 308 & 266 & 231 & 190 & 168 \\ \text { CZP } & 229 & 164 & 142 & 131 & 101 & 77 & 50 & 26 \\ \text { ETN } & 891 & 694 & 629 & 580 & 531 & 476 & 418 & 384 \\ \text { GLM } & 464 & 342 & 296 & 239 & 191 & 162 & 138 & 120 \\ \text { IFX } & 748 & 641 & 580 & 501 & 440 & 390 & 352 & 322 \\ \text { TCZ } & 895 & 709 & 613 & 548 & 461 & 412 & 350 & 302\end{array}$

Number at risk

$\begin{array}{lllllllll}\text { ABT } & 632 & 512 & 454 & 399 & 351 & 284 & 223 & 175 \\ \text { ADA } & 458 & 360 & 308 & 257 & 228 & 199 & 166 & 145 \\ \text { CZP } & 214 & 155 & 135 & 124 & 95 & 72 & 46 & 23 \\ \text { ETN } & 729 & 577 & 523 & 484 & 443 & 401 & 352 & 321 \\ \text { GLM } & 434 & 329 & 286 & 233 & 188 & 160 & 136 & 118 \\ \text { IFX } & 564 & 487 & 438 & 374 & 331 & 288 & 257 & 232 \\ \text { TCZ } & 752 & 623 & 536 & 477 & 397 & 353 & 296 & 251\end{array}$

Fig. 1 Drug survival rates due to lack of effectiveness in a non-adjusted cases and $\mathbf{b}$ adjusted cases. Adjusted confounders were baseline sex, age, disease duration, concomitant prednisolone and methotrexate, and number of previously used bDMARDs. $\mathrm{ABT}=\mathrm{abatacept}$, ADA = adalimumab, CZP = certolizumab pegol, ETN = etanercept, GLM = golimumab, IFX = infliximab, TCZ = tocilizumab, bDMARDs = biological disease-modifying antirheumatic drugs 


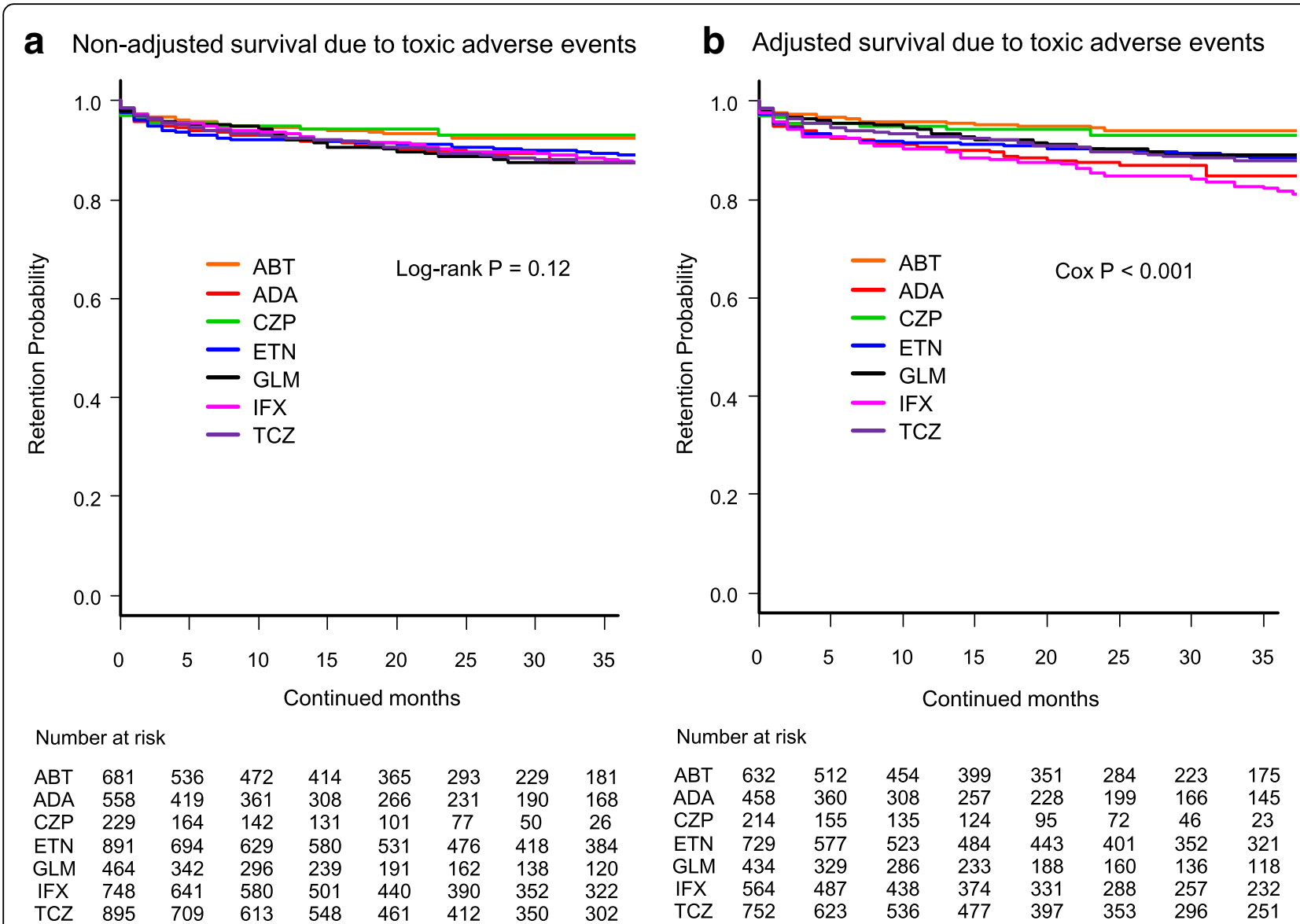

Fig. 2 Drug survival rates due to toxic adverse events in a non-adjusted cases and $\mathbf{b}$ adjusted cases. Adjusted confounders were baseline sex, age, disease duration, concomitant prednisolone and methotrexate, and number of previously used bDMARDs. ABT = abatacept, $A D A=$ adalimumab, CZP = certolizumab pegol, ETN = etanercept, GLM = golimumab, IFX = infliximab, TCZ = tocilizumab, bDMARDs = biological disease-modifying antirheumatic drugs 